\title{
Temperature Dependence of Electrical and Optical Modulation Responses of Quantum-Well Lasers
}

\author{
T. Keating, X. Jin, S. L. Chuang, Fellow, IEEE, and K. Hess, Fellow, IEEE
}

\begin{abstract}
We present theory and experiment for high-speed optical injection in the absorption region of a quantum-well laser and compare the results with those of the electrical injection including carrier transport effect. We show that the main difference between the two responses is the low-frequency roll-off. By using both injection methods, we obtain more accurate and consistent measurements of many important dynamic laser parameters, which include the differential gain, carrier lifetime, $K$ factor, and gain compression factor. Temperature-dependent data of the test laser are presented which show that the most dominant effect is the linear degradation of differential gain and injection efficiency with increasing temperature. While the $K$-factor is insensitive to temperature variation for multiple-quantum-well lasers, we find that the carrier capture time and nonlinear gain suppression coefficient decreases as temperature increases.
\end{abstract}

Index Terms-Differential gain, electrical modulation, optical injection, transport effect.

\section{INTRODUCTION}

$\mathbf{H}$ IGH-SPEED modulation of long-wavelength semiconductor lasers is of major technological importance. The continuing development of high-bandwidth fiber optical communication systems and the unceasing demand for greater data transmission capacity over fiber-optic cables is dependent on the superior qualities offered by semiconductor laser optical transmitters. Long-wavelength lasers, as studied in this paper, are especially useful for long-haul communications since the loss in optical fibers is minimal near wavelengths of $1.55 \mu \mathrm{m}$. Practical communication systems based on these lasers also require robust temperature performance for application in ambient, uncooled environments where the device may be required to operate between $-40{ }^{\circ} \mathrm{C}$ and $85{ }^{\circ} \mathrm{C}$. To improve these devices, it is therefore important to study their temperature-dependent response and analyze which effects are most responsible for limiting the modulation bandwidth.

The excessive damping that limits the electrical modulation bandwidth of quantum-well (QW) lasers originates from a number of possible mechanisms, including photon lifetime [1], carrier capture and escape [2], [3], carrier diffusion [4], carrier heating [5], [6], spectral hole burning [7], and circuit parasitics [8]. The photons generated by the stimulated recombination

Manuscript received March 9, 1999; revised June 16, 1999. This work was supported by the Office of Naval Research under Grant N00014-96-1-0303 and Grant N00014-98-1-0604.

T. Keating, X. Jin, and S. L. Chuang are with the Department of Electrical and Computer Engineering, University of Illinois at Urbana-Champaign, Urbana, IL 61801 USA.

$\mathrm{K}$. Hess is with the Department of Electrical and Computer Engineering and the Beckman Institute, University of Illinois at Urbana-Champaign, IL 61081 USA.

Publisher Item Identifier S 0018-9197(99)07609-5. of electrons and holes travel in the cavity. On average, they exist for a certain amount of time and disappear from the cavity due to the intrinsic absorption or transmission from the mirror facets. Therefore, the photon lifetime constitutes an intrinsic limitation on the modulation response. The major sources of carrier heating in semiconductor lasers are injection heating, stimulated recombination heating, and free carrier heating. These changes in the carrier temperature will be reflected in the changes in gain. An increase of a few degrees will result in a decrease in gain of several percent, which appears in the rate equations as a nonlinear gain suppression coefficient. Spectral hole burning theory also predicts an increase in the nonlinear gain suppression coefficient with increasing carrier temperature. Parasitic effects come from the bias circuit and the shunting of modulation current around the active layer, which will cause a low-frequency roll-off of modulation response. The carrier diffusion, capture, and escape times are usually defined to characterize the carrier transport processes [9], which give a parasitic-like roll-off and are indistinguishable from parasitic effects. In QW lasers, the carrier transport effect is an important limit for multiplequantum-well (MQW) laser modulation bandwidth. In general when the number of wells increases, the modulation bandwidth of the device initially increases, but it is ultimately saturated by the carrier transport effect. This saturation effect was observed in gain-coupled InGaAsP distributed feedback (DFB) lasers with more than eight QW's [10].

A schematic of high-speed modulation by electrical injection and optical injection is shown in Fig. 1. For electrical modulation, the electrons are injected from the outer edge of the left separate-confinement-heterostructure $(\mathrm{SCH})$ region and the holes from the outer edge of the right $\mathrm{SCH}$ region. The injected carriers diffuse through the $\mathrm{SCH}$ region and are captured into the QW's before recombining by stimulated emission processes. Compared with electrical modulation, optical modulation with an optical energy in the absorption range of the QW directly produces photon-generating carriers inside the test laser's active region via injection of a modulated laser beam through one of the test laser's mirror facets. Therefore, the majority of carriers transporting through the $\mathrm{SCH}$ region is not required for lasing action although the coupling between $\mathrm{SCH}$ and QW states still exists for optical modulation. In this way, optical modulaton removes the severe low-frequency roll-off due to the transport and parasitic effects and helps to clarify the intrinsic response.

In this paper, the high-speed modulation response of a $\mathrm{QW}$ DFB laser operating at $1.55 \mu \mathrm{m}$ is investigated for two types of modulation, electrical and optical. Electrical modulation 


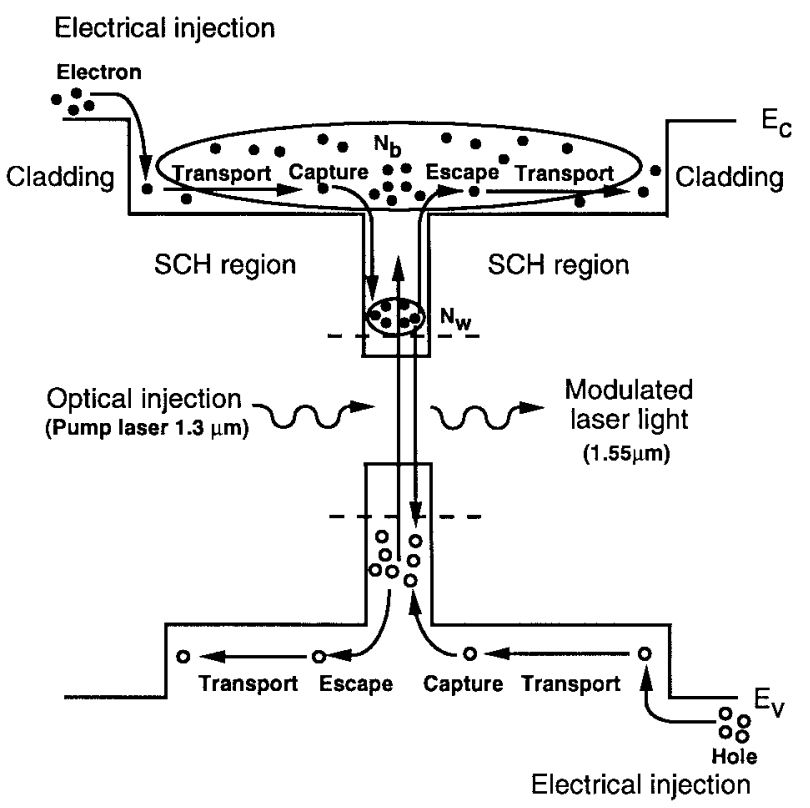

Fig. 1. Energy-band QW potential diagram for high-speed modulation by optical or electrical injections. For electrical injection, the carriers have to transport and get captured by the QW's. For optical injection, we choose the optical pump energy such that carriers are generated in the wells.

is performed by applying a microwave signal directly on the injection current of the test laser. Optical modulation is performed by injecting light from a second pump laser operating at $1.3 \mu \mathrm{m}$ which itself is being directly modulated electrically, so that the injected light is absorbed in the QW's of the 1.55- $\mu \mathrm{m}$ test laser. Both sets of responses are compared with theory in order to extract the values of the fundamental parameters which determine as well as limit the modulation bandwidth. Optical injection at this wavelength eliminates circuit parasitic and carrier transport effects, and the intrinsic laser response can be directly measured [11], [12]. Our experiment differs from the experiment performed in [11], where the optical injection is at a wavelength close to that of the test laser. Therefore, competition of carriers and gain saturation have to be considered for both the pump and test wavelengths. We inject pump signal into the center of the active waveguide region of the test laser to avoid the spatial effects on the optical modulation and optimize the modulation response. It has been shown [14] that the optical modulation response can be influenced by the spatial characteristics (the spot size and its position) of the pump light. Our setup is similar to that in [15], where the intrinsic absorption of the pump light modulates the test laser output. However, little work has been performed so far on directly comparing the electrical and optical modulation response at different temperatures. We show data for temperature dependence of both optical and electrical injection. To analyze the basic parameters controlling the modulation response, the simplest approach is to use rate equations for the cavity photon density and modulated carrier density. Rate equations provide a reasonable description of most of the observed phenomena.

This paper is organized as follows. In Section II, the optical modulation theory is presented and compared with that of electrical modulation including transport effects. In Section III, our experimental setup is described and comparisons between optical and electrical modulation responses are made, and the extracted high-speed parameters are shown. Finally, the temperature dependence of important laser parameters are discussed in Section IV.

\section{RATE EQUATIONS FOR OPTICAL AND ElECTRICAL MODULATION}

Fig. 1 shows a typical SCH QW laser. We will model the carrier transport (diffusion, capture, and emission) in such a laser structure and study its effects on the small-signal modulation with electrical injection and optical injection. Our objective is to calculate the small-signal frequency response of the test laser for optical modulation from the pump laser and use the same rate equations with a different source term to derive the electrical modulation response of the test laser. In general, transport effects for electrical microwave modulation can be modeled by taking into account the carrier density in the $\mathrm{SCH}$ region, the carrier density in the well region, and the photon density separately [9]. Coupling of the carrier density in the barrier states above the QW's to the carrier density in the QW's is modeled by two terms representing carrier capture and escape into or from the wells, respectively. In this case, three rate equations are needed. The source term enters through the injection current in electrical modulation. The model considers carrier injection from the outer edges of the $\mathrm{SCH}$ region, diffusion across the $\mathrm{SCH}$ region, and the subsequent capture and emission of carriers by the QW. For optical injection using an external pump laser, the pump photon density $S_{p}$ acts as the source term. Since the optical energy of the pump laser determines whether the photons are absorbed in the well or in the barriers, we choose the pump wavelength to be longer than the bandgap wavelength of the barriers and shorter than the bandedge wavelength of the wells so that absorption occurs only in the wells. The rate equations for both cases are written as

$$
\begin{aligned}
\frac{d N_{b}}{d t} & =\frac{\eta_{\mathrm{inj}} I}{q V_{b}}-\frac{N_{b}}{\tau_{b}}-\frac{N_{b}}{\tau_{b w}}+\frac{N_{w} V_{w}}{\tau_{w b} V_{b}} \\
\frac{d N_{w}}{d t} & =\frac{N_{b} V_{b}}{\tau_{b w} V_{w}}-\frac{N_{w}}{\tau_{w}}-\frac{N_{w}}{\tau_{w b}}-\frac{v_{g} G\left(N_{w}\right) S}{1+\epsilon S}+v_{g} \alpha_{p} S_{p} \\
\frac{d S}{d t} & =\frac{\Gamma v_{g} G\left(N_{w}\right) S}{1+\epsilon S}-\frac{S}{\tau_{p}}
\end{aligned}
$$

where $q$ is the electron unit charge, $G\left(N_{w}\right)$ is the optical gain at the carrier concentration $N_{w}$ in the bound states of the wells, $N_{b}$ is the carrier density in the barrier (continuum) states including the $\mathrm{SCH}$ and active layers, $S$ is the photon density of the test laser, $\epsilon$ is the nonlinear gain suppression coefficient, $\Gamma$ is the optical confinement factor, $\tau_{p}$ is the photon lifetime, $\eta_{\text {inj }}$ is the injection efficiency, $I$ is the injection electrical current, $V_{b}$ is the volume of the $\mathrm{SCH}$ and active region, $V_{w}$ is the volume of the well region, $\tau_{b}$ is the carrier recombination lifetime in the barrier region, $\tau_{w}$ is the carrier recombination lifetime in the well, $\tau_{b w}$ is the effective carrier diffusion across the SCH region and capture time by the wells, and $\tau_{w b}$ is the thermionic emission and carrier diffusion time from the well to the barrier states. The final term in (2) is a source term which 
represents the photon generation due to optical pumping at the wavelength $(1.3 \mu \mathrm{m})$ where the optically injected photons are absorbed in the well regions. $S_{p}$ is the pump photon density, and $\alpha_{p}$ is the absorption at the pump wavelength. The above model is often referred to as the reservoir model [9] and is equivalent to models which incorporate additional effects such as diffusive transport [14]. A spontaneous emission term has been ignored in (3) for above threshold operation.

Assuming a small-signal optical injection due to the external pump laser

$$
S_{p}(t)=S_{p 0}+s_{p}(\omega) e^{j \omega t}
$$

where the test laser is biased at a dc current $I(t)=I_{0}$ above threshold. The responses can be solved by assuming

$$
\begin{aligned}
S(t) & =S_{0}+s(\omega) e^{j \omega t} \\
N_{b}(t) & =N_{b 0}+n_{b}(\omega) e^{j \omega t} \\
N_{w}(t) & =N_{w 0}+n_{w}(\omega) e^{j \omega t}
\end{aligned}
$$

and

$$
G\left(N_{w}\right)=G\left(N_{w 0}\right)+g^{\prime}\left(N_{w}-N_{w 0}\right)
$$

where $g^{\prime}$ is the differential gain of the test laser, and $N_{w o}$ is steady-state carrier density in the QW.

Substituting (4)-(8) into (1)-(3), the steady-state quantities can be obtained by setting the time-dependent terms to zero. Then, the small-signal equations for the time-dependent terms give

$$
\begin{aligned}
j \omega n_{b}= & 0-\frac{n_{b}}{\tau_{b}}-\frac{n_{b}}{\tau_{b w}}+\frac{n_{w} V_{w}}{\tau_{w b} V_{b}} \\
j \omega n_{w}= & \frac{n_{b} V_{b}}{\tau_{b w} V_{w}}-\frac{n_{w}}{\tau_{w}}-\frac{n_{w}}{\tau_{w b}}-v_{g}\left[\frac{G_{0} s}{1+\epsilon S_{0}}-\frac{G_{0} S_{0} \epsilon s}{\left(1+\epsilon S_{0}\right)^{2}}\right. \\
& \left.+\frac{g^{\prime} S_{0} n_{w}}{1+\epsilon S_{0}}\right]+v_{g} \alpha_{p} S_{p} \\
j \omega s= & \Gamma v_{g}\left[-\frac{G_{0} S_{0} \epsilon s}{\left(1+\epsilon S_{0}\right)^{2}}+\frac{g^{\prime} S_{0} n_{w}}{1+\epsilon S_{0}}\right]
\end{aligned}
$$

where the steady-state gain-loss relations $\Gamma v_{g} G_{0} /\left(1+\epsilon S_{0}\right)=$ $1 / \tau_{p}$, and the Taylor expansion $1 /(1+\epsilon S)=1 /\left(1+\epsilon S_{0}\right)-$ $\epsilon s /\left(1+\epsilon S_{0}\right)^{2}$ are used. This set of small-signal equations can be reduced by eliminating $n_{b}$ and $n_{w}$ to give a relationship shown in (12), at the bottom of the page, between the test laser signal $s(\omega)$ and the pump signal $s_{p}(\omega)$.

The optical modulation response can be written in a normalized form

$$
\frac{M(\omega)}{M(0)}=\frac{\omega_{r}^{2}}{\omega_{r}^{2}-\omega^{2}+j \omega \gamma} \quad \text { (optical) }
$$

where

$$
\begin{aligned}
\omega_{r}^{2} & =\frac{\left(v_{g} g^{\prime} / \chi\right) S_{0}}{\tau_{p}\left(1+\epsilon S_{0}\right)}\left(1+\frac{\epsilon}{v_{g} g^{\prime} \tau_{w}}\right) \\
\gamma & =\frac{\left(v_{g} g^{\prime} / \chi\right) S_{0}}{1+\epsilon S_{0}}+\frac{\epsilon S_{0}}{\left(1+\epsilon S_{0}\right) \tau_{p}}+\frac{1}{\chi \tau_{w}} \\
\chi & =1+\frac{\tau_{b w}}{\tau_{w b}} .
\end{aligned}
$$

For a small-signal electrical injection, $I(t)=I_{0}+i(\omega) e^{j \omega t}$, with no external pump, $S_{p}(t)=0$. The resulting modulation response is given by [9] and [21] in (17), shown at the bottom of the page, which can be written in the form

$$
\frac{M(\omega)}{M(0)}=\frac{\omega_{r}^{2}}{\left(1+j \omega \tau_{b w}\right)\left(\omega_{r}^{2}-\omega^{2}+j \omega \gamma\right)} \quad \text { (electrical) }
$$

where $\tau_{b w}$ is a constant which contributes to low-frequency roll-off. The interpretation of this effect is not straightforward. The roll-off in the modulation response derived above is equivalent to the roll-off caused by a simple electrical parasitic, such as a capacitance in parallel to the device. However, in real devices, the effective capacitance may be bias-dependent since the device capacitance is related to the storage of charge in the forward-biased junction. The charge storage itself results from a combination of effects including transport and carrier heating and is difficult to model in terms of a small number of rate equations [15]-[17]. Despite these difficulties, the modulation response can be matched very well for the experiments here with a single total roll-off frequency $f_{p}$ for a given temperature. If the transport effect dominates, the rolloff frequency of the transport effect is $f_{p}=1 /\left(2 \pi \tau_{b w}\right)$. In this case, we can measure the carrier diffusion and capture time of the QW.

Because the stimulated emission occurs in the QW's, the carrier lifetime in the $\mathrm{SCH}$ or barrier region is usually very

$$
\begin{aligned}
M(\omega) & =\frac{s(\omega)}{s_{p}(\omega)} \\
& =\frac{V_{g}^{2} \alpha_{p} \Gamma g^{\prime} S_{0}}{\left[j \omega\left(1+\frac{j \omega \tau_{b w}+\tau_{b w} / \tau_{b}}{j \omega \tau_{w b}\left(j \omega \tau_{b w}+1+\tau_{b w} / \tau_{b}\right)}\right)+\frac{1}{\tau_{w}}+\frac{S_{0} g^{\prime} v_{g}}{1+\epsilon S_{0}}\right]\left(j \omega+\frac{\epsilon S_{0}}{\tau_{p}\left(1+\epsilon S_{0}\right)}\right)+\frac{v_{g} g^{\prime} S_{0}}{\tau_{p}\left(1+\epsilon S_{0}\right)^{2}}}
\end{aligned}
$$

$$
\begin{aligned}
M(\omega) & =\frac{s(\omega)}{i(\omega)} \\
& =\frac{\frac{\eta_{i n j}}{q V_{w}}}{\left(1+j \omega \tau_{b w}\right)} \frac{V_{g} \Gamma g^{\prime} \frac{S_{0}}{\left(1+\epsilon S_{0}\right)}}{\left\{\left[j \omega\left(1+\frac{\tau_{b w}}{\tau_{w b}\left(j \omega \tau_{b w}+1\right)}\right)+\frac{1}{\tau_{w}}+\frac{S_{0} g^{\prime} v_{g}}{1+\epsilon S_{0}}\right]\left[j \omega+\frac{\epsilon S_{0}}{\tau_{p}\left(1+\epsilon S_{0}\right)}\right]+\frac{v_{g} g^{\prime} S_{0}}{\tau_{p}\left(1+\epsilon S_{0}\right)^{2}}\right\}}
\end{aligned}
$$


TABLE I

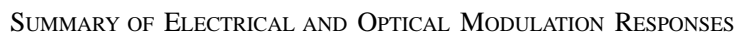

\begin{tabular}{c}
\hline Electrical Pumping and $1.3 \mu \mathrm{m}$ Optical Pumping \\
\hline $\begin{array}{c}M(\omega) \\
M(0)\end{array}=\frac{M(\omega)}{\left(1+j \omega \tau_{b w}\right)\left(\omega_{r}^{2}-\omega^{2}+j \omega \gamma\right)} \quad \frac{M(\omega)}{M(0)}=\frac{\omega_{r}^{2}}{\omega_{r}^{2}-\omega^{2}+j \omega \gamma}$ \\
$\gamma=\frac{1}{\tau_{w} \chi}+\frac{v_{g} g^{\prime} S_{0}}{\tau_{p} \chi\left(1+\epsilon S_{0}\right)}\left(\tau_{p}+\frac{\epsilon \chi}{v_{g} g^{\prime}}\right)$ \\
$\omega_{r}^{2}=\frac{S_{0} v_{g} g^{\prime}}{\tau_{p} \chi\left(1+\epsilon S_{0}\right)}\left(1+\frac{\epsilon}{\tau_{w} v_{g} g^{\prime}}\right)$ \\
$\chi=1+\frac{\tau_{b w}}{\tau_{w b}}$ \\
\hline
\end{tabular}

long, so we set $\tau_{b w} / \tau_{b} \ll 1$ in (12). The modulation responses for both injections are summarized in Table I. Thus the comparison between the optical and electrical modulations is clear. The relaxation frequency $\omega_{r}=2 \pi f_{r}$ and damping factor $\gamma$ for both cases are the same. The transport factor $\chi=1+\tau_{b w} / \tau_{w b}$ has also been introduced to the equations. The only difference between the two responses is that optical injection has no low frequency roll-off, which corresponds to transport effects. There would also be a difference when the number of wells is varied. Thus, optical modulation gives an intrinsic modulation response of a laser since the injected pump light is chosen such that the pump light is absorbed only in the well region.

For optical injection, the carriers are injected directly into the QW region, which removes the requirement of carrier transport from the $\mathrm{SCH}$ region to the $\mathrm{QW}$ region before lasing. In other words, optical injection removes the lowfrequency roll-off caused by carriers transporting through $\mathrm{SCH}$ region. However, the $\mathrm{SCH}$ and QW regions are still coupled together. There are always carriers being captured by and escaping from the QW's. The rate of capture and escape will influence the modulation response intrinsically. Therefore, because of the SCH structure, carrier diffusion capture-escape still affects the optical modulation response, which comes in through the $\chi$ factor. Thus, the effective differential gain is reduced by a factor of $\chi$ for the same photon density. This reduction is present even in the absence of low-frequency rolloff in the optical injection, which results in the reduction of the resonance frequency. The effective carrier recombination lifetime in the well is also increased by a factor of $\chi$, and the nonlinear gain suppression coefficient remains unchanged. Another important factor in high-speed modulation is the $K$ factor, which is the slope of the damping factor versus relaxation frequency squared

$$
\begin{aligned}
& \gamma=K f_{r}^{2}+\frac{1}{\chi \tau_{w}} \\
& K=4 \pi^{2}\left(\tau_{p}+\frac{\epsilon}{v_{g} g^{\prime} / \chi}\right) .
\end{aligned}
$$

The $K$ factor is usually used to determine the maximum possible modulation bandwidth $f_{\max }=2 \pi \sqrt{2} / K$. A small $K$ factor means a large laser bandwidth. Even if we use optical injection modulation, the carrier capture and escape processes still increase the $K$ factor and limit the maximum modulation. The above parameters characterize the intrinsic modulation response of semiconductor lasers.
TABLE II

Structure of the Test LASER

\begin{tabular}{lllll}
\hline Well: & Number of wells & 7 & SCH width: & $705 \AA$ \\
& Material & InGaAsP & Stripe width: & $1.2 \mu \mathrm{m}$ \\
& Strain & $-1.6 \%$ comp. & Cavity length: & $400 \mu \mathrm{m}$ \\
& Width & $70 \AA$ & & \\
& PL wavelength & $1.5564 \mu \mathrm{m}$ & & \\
\hline Barrier: & Material & InGaAsP & & \\
& Strain & lattice-matched & \\
& Width & $100 \AA$ & & \\
& PL wavelength & $1.255 \mu \mathrm{m}$ & \\
\hline
\end{tabular}

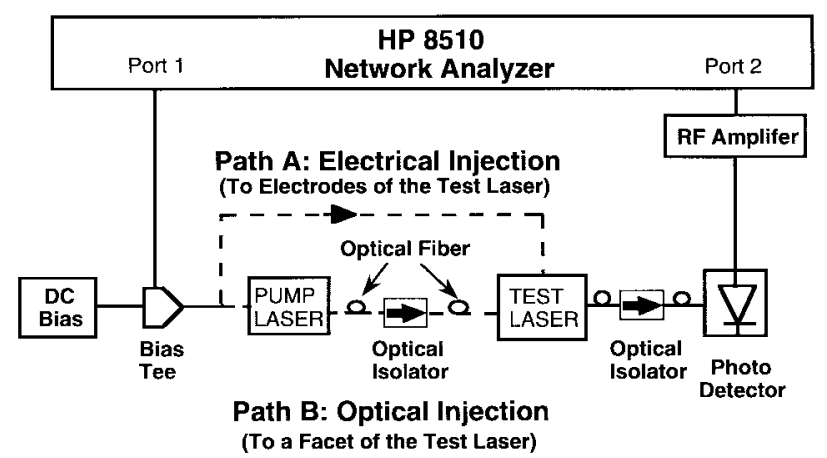

Fig. 2. Schematic diagram of electrical and optical modulation experiment. Path A is for electrical injection experiment. Path B is for optical injection experiment with a $1.3-\mu \mathrm{m}$ pump.

\section{EXPERIMENTAL RESULTS}

The high-speed modulation response of a buried heterostructure MQW DFB laser was measured. The composition of the undoped active region is described in Table II. The barrier photoluminescence wavelength peak occurs at $1.255 \mu \mathrm{m}$. Therefore, pump light at $1.3 \mu \mathrm{m}$ will be absorbed in the QW's.

A schematic diagram of the modulation response experiments is shown in Fig. 2. The laser is held at constant temperature and measurements are made at $20^{\circ} \mathrm{C}, 25^{\circ} \mathrm{C}, 30^{\circ} \mathrm{C}$, $35^{\circ} \mathrm{C}$, and $40{ }^{\circ} \mathrm{C}$. In the electrical modulation experiments, shown as path A in Fig. 2, the HP 8510 network analyzer provides a small microwave signal $(0 \mathrm{dBm})$ at frequencies swept from $45 \mathrm{MHz}$ to $10 \mathrm{GHz}$, which is coupled to the test laser electrodes through a bias-T and a high-speed probe. The laser light is coupled to a fiber using a lensed fiber-optical interface and travels through an optical isolator before being measured by a high-speed $(29 \mathrm{GHz})$ photodetector. The smallsignal response is increased by an 18-dB-gain RF amplifier before entering the network analyzer, which measures the magnitude of the modulation response $|M(\omega) / M(0)|^{2}$. The data are averaged to reduce noise. The optical response measurements shown as path B in Fig. 2 are similar to electrical modulation experiments, except that a $1.3-\mu \mathrm{m}$ pump laser with a wide bandwidth is modulated electrically, and its modulated optical output is injected into the test DFB laser facet. The modulation of the pump laser is recorded at a fixed bias and stored for the calibration of the optical response of the $1.55-\mu \mathrm{m}$ test laser. The pump light is completely absorbed in the test laser, so the light coupled out of the test laser does not require additional filtering of the pump light. 


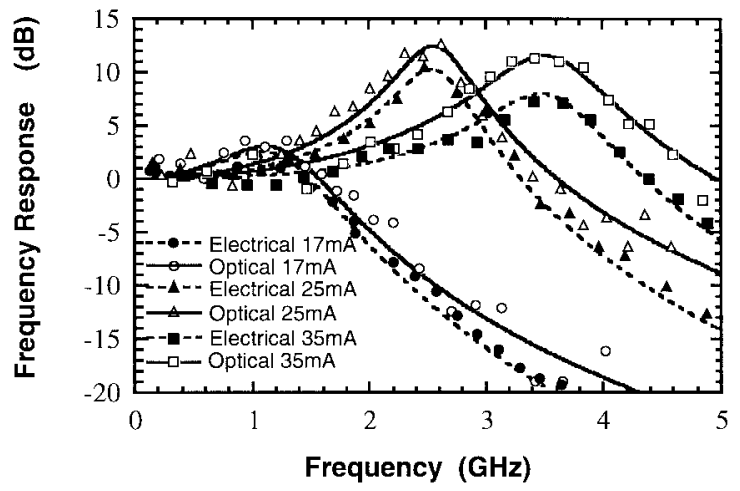

Fig. 3. Normalized modulation response, $10 \log \left(|M(\omega) / M(0)|^{2}\right)$, due to electrical injection and optical injection at $1.3 \mu \mathrm{m}$. The solid and dashed lines are theoretical modulation responses using (13) and (18), and symbols are measurement results.

The electrical and optical modulation responses are first compared at a fixed temperature of $20{ }^{\circ} \mathrm{C}$. Fig. 3 shows the least-square fits of the measured modulation responses using the theory derived above for electrical and optical injections. The fits are used to extract the differential gain and nonlinear gain suppression factor. In general, the optical injection measurements exhibit more noise than the electrical injection measurements, since the system losses are higher for the optical injection experiments. However, the optical modulation responses have less parameters to fit, which means less freedom of choosing a number. On the other hand, the electrical modulation responses usually have a higher signalto-noise ratio and more parameters to be handled than the optical modulation. Thus, simultaneously fitting electrical and optical modulation responses can compensate each method's shortage and obtain more accurate laser parameters. Also, by comparing the two responses, the roll-off frequency $f_{p}$ can be obtained more accurately. Both modulation responses show clearly that the relaxation peaks increase in frequency as the $\mathrm{dc}$ bias is increased. The modulation responses due to optical injection exhibit a slower roll-off at high frequencies, which indicates the absence of low-frequency pole on the optical response. Also, the peak of the optical pump response is generally higher than that of the electrical, and the differences between the two responses increase with increasing current. For $35-\mathrm{mA}$ current injection, electrical modulation has very obvious roll-off because the roll-off frequency is $3.52 \mathrm{GHz}$ at this temperature $\left(20^{\circ} \mathrm{C}\right)$. Fig. 4 shows that the roll-off frequency $f_{p}$ at various temperatures from $20^{\circ} \mathrm{C}$ to $40^{\circ} \mathrm{C}$ is independent of the current bias. As we discussed before, the shunt capacitance of parasitic effects may be bias-dependent, and the roll-off frequency of the transport effect is only determined by carrier diffuse-capture time $\tau_{b w}$. Although we cannot exclude the parasitic effects, we still can see that the transport effects are more important than the parasitic effects for this DFB laser.

In Fig. 5(a), the relaxation frequency squared is plotted versus optical power. The slope of the linear fit will be used later to extract the differential gain. The damping factor is calculated using extracted parameters from the modulation responses and is plotted in Fig. 5(b). The slope of the linear fit at large relaxation frequency is the $K$ factor. The deviation at

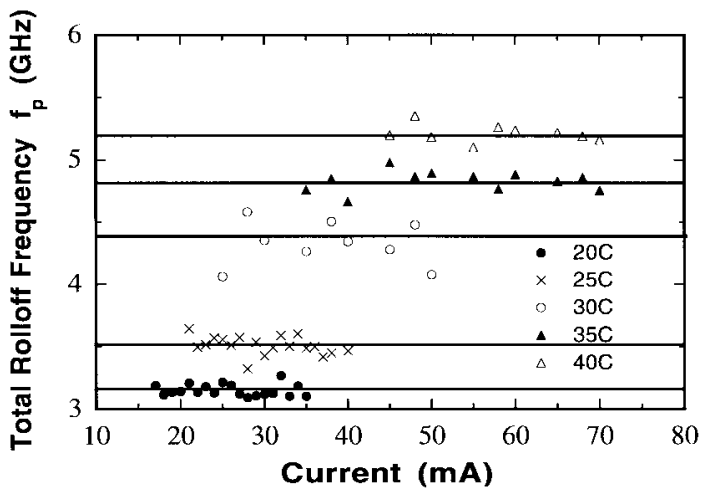

Fig. 4. The total roll-off frequency $\left(f_{p}=1 / 2 \pi \tau_{b w}\right)$ versus the bias current at different temperature. The solid line is the average value of roll-off frequency.

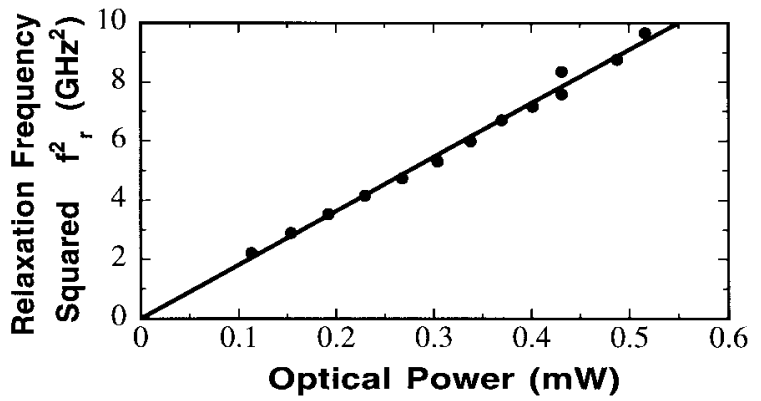

(a)

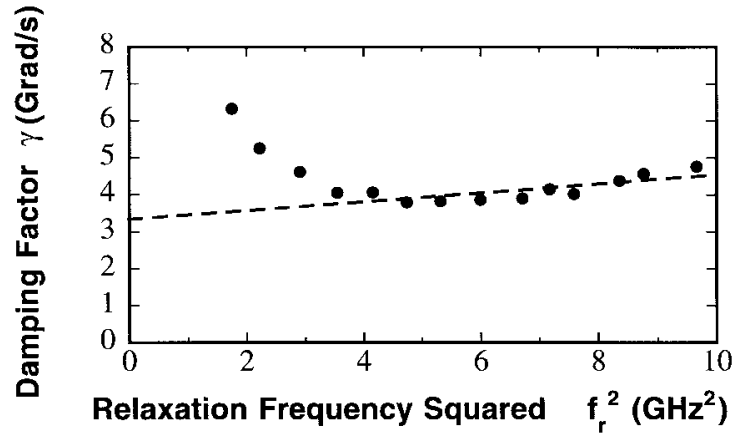

(b)

Fig. 5. (a) The relaxation frequency squared versus optical power at $20^{\circ} \mathrm{C}$. The line is the least-square fit to the data using (14). (b) The damping factor versus the relaxation frequency squared at $20^{\circ} \mathrm{C}$. The dash line is the linear fit for large relaxation frequency, with a slope equal to the $K$ factor.

the low-frequency end between the data and the linear dashed line is due to the negligence of the spontaneous emission term. If we include the spontaneous emission factor $R_{\mathrm{sp}}$, the damping factor should have an additional term $R_{\mathrm{sp}} / S$ which can be neglected at high photon density $S$ (corresponding to a large relaxation frequency). The complete set of extracted parameters at $20^{\circ} \mathrm{C}$ is listed in Table III. The differential gain and nonlinear gain suppression coefficient are well within the values typically found in the literature.

\section{TEMPERATURE DEPENDENCE}

It is important to determine which factors affecting the modulation responses are most sensitive to temperature. The 
TABLE III

High-Speed Laser Parameters and Extracted Values at $20^{\circ} \mathrm{C}$, Using Simultaneous Fitting of the Electrical and Optical Modulation Responses

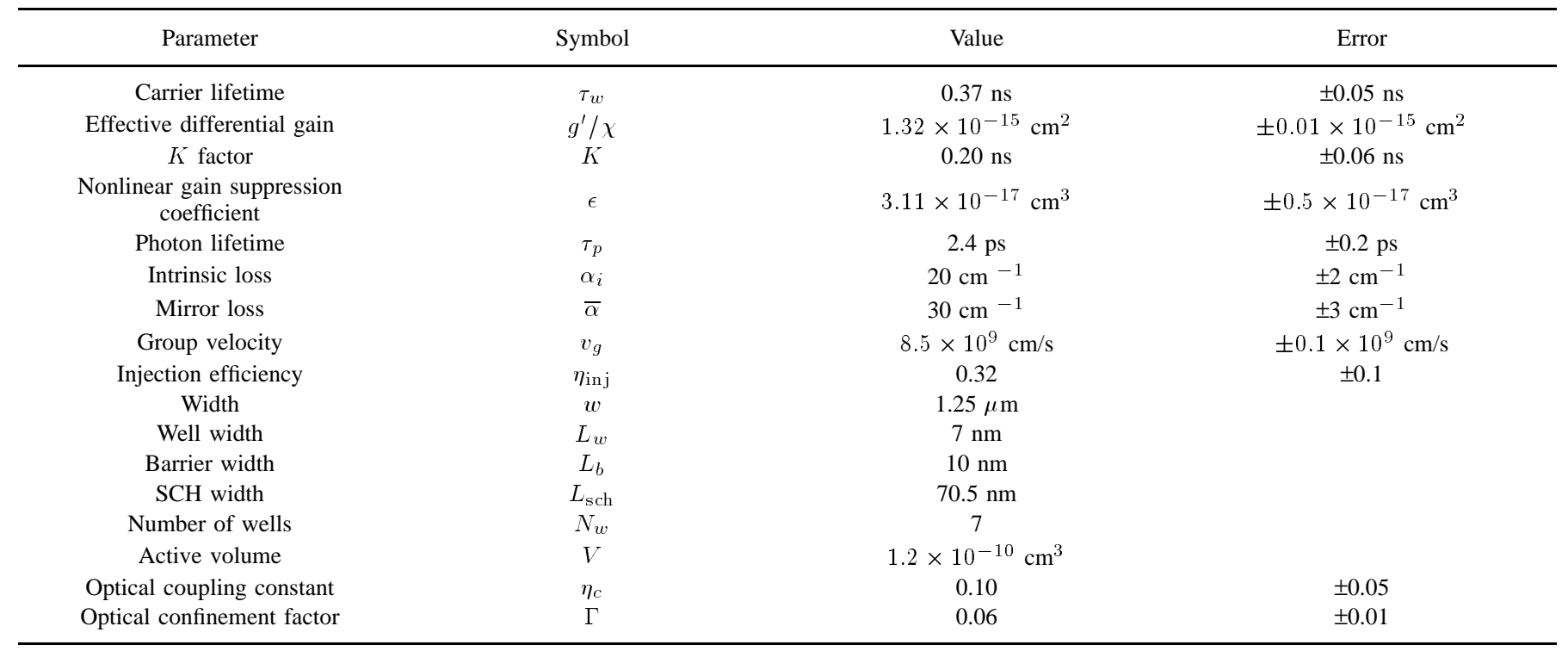

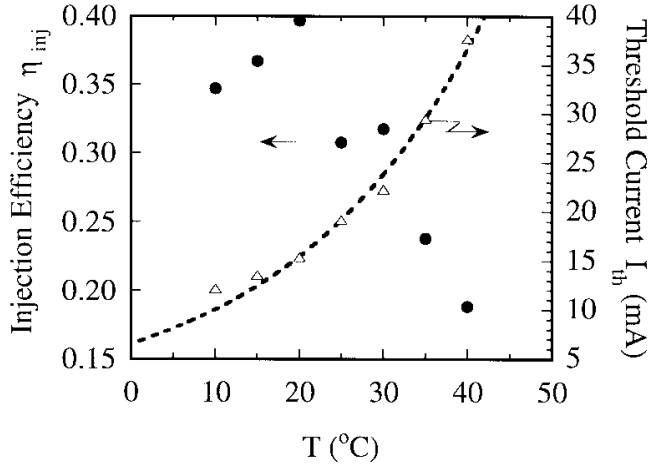

Fig. 6. The injection efficiency and threshold current as a function of temperature. The efficiency degrades severely with temperature. The solid line shows the trend. The dashed line is fitting of threshold current using the formula $I_{\mathrm{th}}=I_{o} e^{T / T_{o}}$, where $T_{o}=24 \mathrm{~K}$.

fitting procedure described above is applied to each set of modulation responses at each temperature. In Fig. 6, the injection efficiency and threshold current of the test laser are plotted versus temperature. This plot is obtained from measured $L-I$ curves. The injection efficiency is defined as the fraction of current above threshold which results in the radiative recombination. A fraction of the injected current in InGaAsP lasers is lost (by nonradiative recombination or carrier leakage) at high temperatures, and this fraction does not contribute to radiative recombination or optical gain. The injection efficiency degrades relatively severely with temperature, by a factor of more than two over the $30^{\circ}$ temperature increase (about 10\% in absolute temperature). The injection efficiency acts to change the modulation response primarily by reducing the overall magnitude of the response. Indirectly, however, the wasted carriers may contribute to the forward bias capacitance, further influencing the modulation response. An exponential temperature dependence of the threshold current is generally used, and the overall characteristic temperature $T_{o}$ is $24 \mathrm{~K}$ for this laser, which indicates a large dependence on temperature.

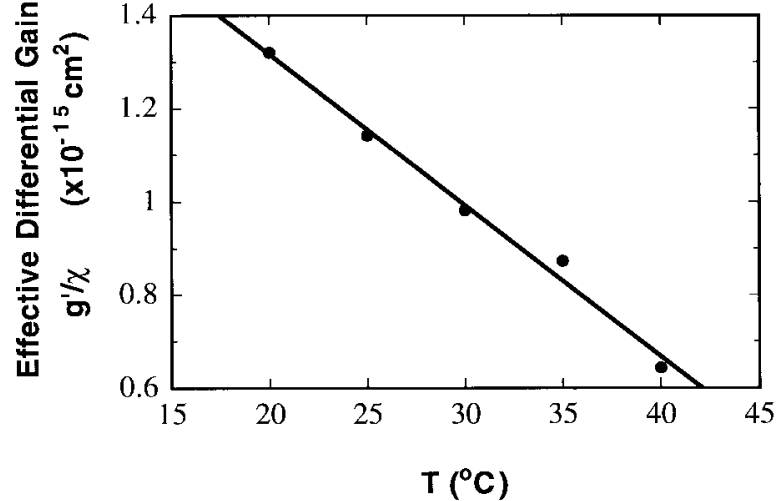

Fig. 7. The effective differential gain $\left(g^{\prime} / \chi\right)$ as a function of temperature, showing strong degradation with temperature. $\chi=1+\tau_{b w} / \tau_{w b}$ accounts for carrier transport/capture effect.

A higher carrier density is necessary to achieve the threshold gain condition with increasing temperature. This results in the nonradiative recombination current being a large fraction of the total current. Several mechanisms have been proposed to explain the observed high-temperature sensitivity of the threshold current of InGaAsP lasers. These are carrier leakage over the heterojunction, Auger recombination, and intervalance band absorption.

The effective differential gain $\left(g^{\prime} / \chi\right)$ also shows a strong temperature dependence and is plotted in Fig. 7. Over the temperature range plotted, the differential gain decreases by a factor of approximately two, which shows a linear function of the temperature [27]. This effect is due to the temperature dependence of the Fermi distribution, the carrier density, and the intrinsic transition linewidth broadening. However, it is difficult to isolate the significance of each quantity on the temperature dependence of a laser because so many physical phenomena are involved. It can be interpreted as follows. The broadening of the Fermi occupation probability function with increasing temperature spreads the carriers over a larger 
energy range for a given overall carrier density. The result is a lower spectral concentration of inverted carriers, which leads to a broadening and flattening of the gain spectrum. Thus, the gain is higher at lower temperatures for the same carrier density, and the carrier density required to achieve a particular gain increases with temperature. In addition, thermionic emission of electrons from the QW to the barrier region is also enhanced at high temperatures. These effects contribute to strong recombination outside the QW and carrier leakage. Since the increased carrier population in the barrier region does not contribute to the optical gain, the differential gain decreases rapidly at high temperatures. This decrease in gain strongly reduces the modulation bandwidth [13]. The differential gain plays a central role in determining the fundamental frequency response of semiconductor lasers since the intrinsic direct modulation speed varies as the square root of the differential gain at the operating carrier density and wavelength.

In Fig. 8(a), the carrier lifetime $\tau_{w}$ in the QW's is plotted and shows a decrease with increasing temperature. The carrier lifetime is expected to shorten with increasing temperature, since in $1.55-\mu \mathrm{m}$ devices the carrier recombination is dominated by Auger recombination [18], which is strongly temperature-dependent. In Fig. 8(b), the total roll-off frequency is plotted versus temperature, showing an increase with increasing temperature. Usually, the low-frequency roll-off is a combination of the effects of the shunt capacitance, external circuit parasitic, and internal transport effect. As for the transport effect, the carrier diffusion and capture time across the $\mathrm{SCH}$ region $\left(\tau_{b w}\right)$ is usually limited by the hole diffusion time, which is insensitive (for MQW's) or slightly increasing (for single-QW's) according to temperature increments from 200 to $350 \mathrm{~K}$ [9], [21]. If we assume the carrier transport effect is dominant, we can calculate the carrier diffusion-capture time $\tau_{b w}$, which is also shown in Fig. 8(b). However, our data shows a decrease of $\tau_{b w}$ with increasing temperature. This can be explained as follows. First, in simple view, the carrier diffusion-capture time $\tau_{b w}$ is inversely proportional to the hole diffusion constant $D=k_{B} T \mu / q$ where the mobility $\mu$ is also temperature-dependent. $k_{B}$ is the Boltzmann constant. Actually the mobility $\mu$ decreases with increasing temperature. The total changes in diffusion constant $D$ are determined by changes in $\mu T$. To be more exact, the carrier diffusion is usually referred to the diffusion capacitance of the test laser, which has a separate temperature dependence not limited to constant D in obvious ways [16]. Second, the transport effect may not play the only role in the rolloff frequency. The electrical parasitic effect can also account for the low-frequency roll-off. However, the external circuit parasitic will not change significantly with laser temperature. Thus, the capacitance of the test laser should respond to the increase of roll-off frequency. This trend indicates that the capacitance decreases with increasing temperature. This is reasonable since the charge storage capacitance decreases with decreasing carrier lifetime. Generally, because of this parasitic frequency, the roll-off frequency cannot be used to directly extract the exact value of carrier diffusion-capture time. Typical values for the well capture time are on the

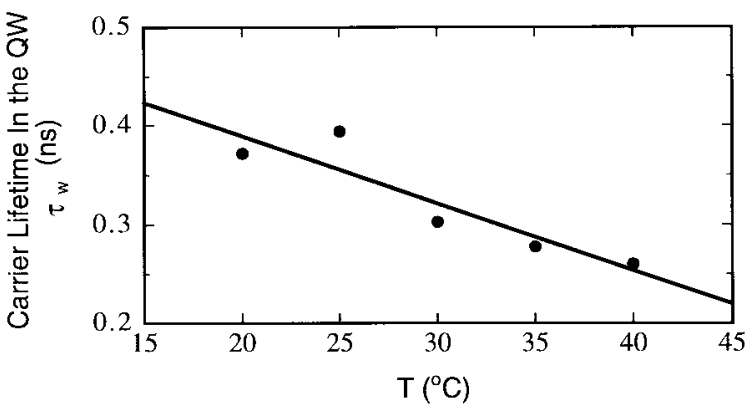

(a)

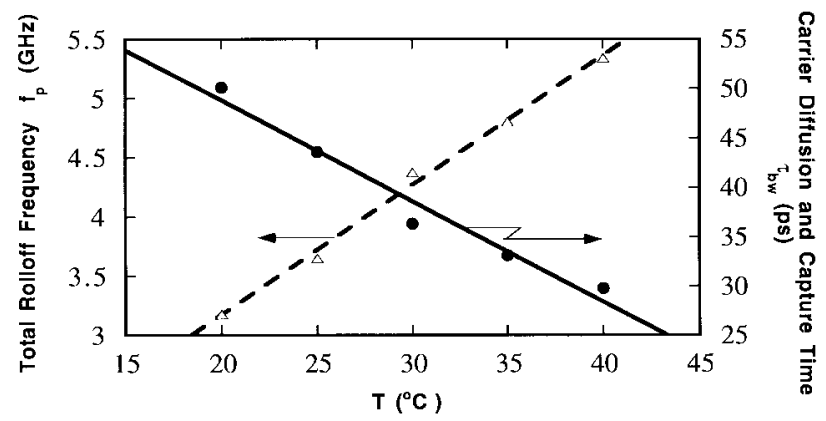

(b)

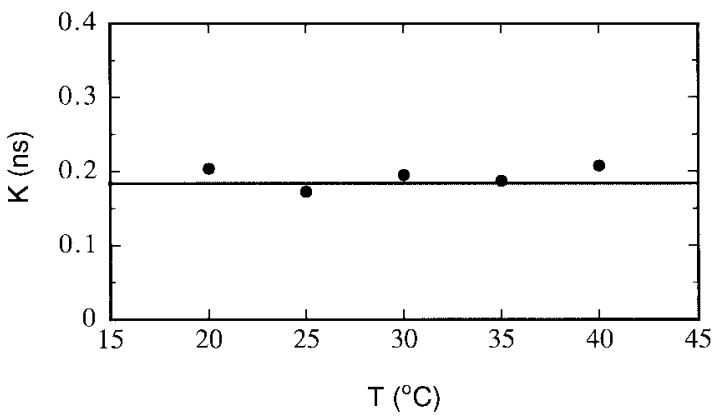

(c)

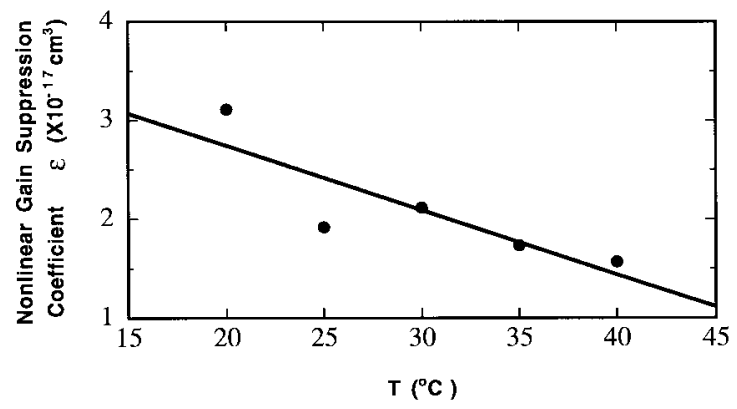

(d)

Fig. 8. (a) The carrier lifetime is plotted as a function of temperature, showing a large decrease as the temperature increases. (b) The roll-off frequency is plotted as a function of temperature, which increases with temperature. The carrier diffusion and capture time is also plotted in (b), which decreases with temperature. (c) The $K$ factor is plotted as a function of temperature, which is insensitive to temperature. (d) The nonlinear gain suppression coefficient is plotted. The line in each figure shows the trend only.

order of tens of picoseconds [9], [14], [21]. Fig. 8(c) shows that the $K$ factor is insensitive to temperature, which agrees with the previous literature [21]. The more QW's there are, the more independent of the $K$ factor on temperature [21]. 
For single-QW lasers, the $K$ factor can vary by two times between 200-350 K [4]. The temperature insensitivity of the $K$ factor implies that the differential gain and the nonlinear gain suppression coefficient have a similar temperature dependence and cancel each other to maintain a temperature-insensitive $\epsilon /\left(g^{\prime} / \chi\right)$ ratio in InGaAsP-InP QW materials. This means the nonlinear gain suppression coefficients should decrease with increasing temperature. The nonlinear gain suppression coefficients of different temperatures are shown in Fig. 8(d), which are particularly sensitive to the extracted value of the damping factor and have large error variations. However, the decrease in nonlinear gain suppression coefficient with increasing temperature still can be seen. The nonlinear gain saturation results from a variety of factors, but it is considered to arise primarily through dynamic carrier heating [5], [6] and perhaps spectral hole burning [7]. If the $K$ factor is constant, it means the maximum bandwidth $f_{\max }$ is insensitive to the temperature. The lower temperature will not improve the potential bandwidth of QW lasers. Indeed, it gets worse because of the lower roll-off frequency. On the other hand, the injection efficiency and high threshold will deteriorate the optical signal at higher temperature. Therefore, designing a high-speed laser is a tradeoff among all aspects.

\section{CONCLUSIONS}

The temperature dependence of the electrical and optical modulation responses have been investigated, and rateequation theoretical models show excellent agreement with the data. The major difference between the two responses is the low-frequency roll-off, which is constant with respect to bias current at a given temperature. The carrier diffusion-captureescape time influences the intrinsic modulation response of QW lasers through the transport factor $\chi$. We fit electrical and optical modulation responses at the same time at each current and each temperature. The combination of two measurements allows us to obtain a set of more accurate laser parameters. Temperature-dependent laser parameters are shown in this paper. When temperature increases, the most dominant effect is the linear degradation of differential gain, and reduction of injection efficiency and carrier lifetime in the QW's. The temperature-dependent response of all parameters closely follows physically reasonable trends.

\section{ACKNOWLEDGMENT}

The authors would like to thank Dr. T. C. Wu for providing expert advice on the optical modulation experiments.

\section{REFERENCES}

[1] C. Y. Tsai, F. P. Shih, T. L. Sung, T. Y. Wu, C. H. Chen, and C. Y Tsai, "A small-signal analysis of the modulation response of high-speed quantum-well lasers: Effects of spectral hole burning, carrier heating, and carrier diffusion-capture-escape," IEEE J. Quantum Electron., vol. 33, pp. 2084-2096, 1997.

[2] S. C. Kan, D. Vassilovski, T. C. Wu, and K. Y. Lau, "On effects of carrier diffusion and quantum capture in high speed modulation of quantum well lasers," Appl. Phys. Lett., vol. 61, pp. 752-754, 1992.

[3] D. Klotzkin, K. Kamath, and P. Bhattacharya, "Quantum capture times at room temperature in high-speed InGaAs-GaAs self-organized quantumdot lasers," IEEE Photon. Technol. Lett., vol. 9, pp. 1301-1303, 1997.
[4] R. Nagarajan, T. Fukushima, S. W. Corzine, and J. E. Bowers, "Effects of carrier transport on high-speed quantum well lasers," Appl. Phys. Lett., vol. 59, pp. 1835-1837, 1991.

[5] M. Grupen and K. Hess, "Severe gain suppression due to dynamic carrier heating in quantum well lasers," Appl. Phys. Lett., vol. 70, pp. 808-810, 1997.

[6] M. P. Kesler and E. P. Ippen, "Subpicosecond gain dynamics in GaAlAs laser diodes," Appl. Phys. Lett., vol. 51, pp. 1765-1767, 1987.

[7] Y. Matsui, H. Murai, S. Arahira, Y. Ogawa, and A. Suzuki, "Enhanced modulation bandwidth for strain-compensated InGaAlAs-InGaAsP MQW lasers," IEEE J. Quantum Electron., vol. 34, pp. 1970-1978, 1998.

[8] R. Olshansky, P. Hill, V. Lanzisera, and W. Powazink, "Frequency response of $1.3 \mu \mathrm{m}$ InGaAsP high speed semiconductor lasers," IEEE J. Quantum Electron., vol. QE-23, pp. 1410-1418, 1987.

[9] R. Nagarajan, M. Ishikawa, T. Fukushima, R. Geels, and J. Bowers, "High speed quantum well lasers and carrier transport effects," IEEE J. Quantum Electron., vol. 28, pp. 1990-2008, 1992.

[10] H. Lu, C. Blaauw, B. Benyon, G. P. Li, and T. Makino, "High-power and high-speed performance of $1.3-\mu \mathrm{m}$ strained MQW gain-coupled DFB lasers," IEEE J. Select. Topics Quantum Electron., vol. 1, pp. 375-381, 1995.

[11] C. B. Su, J. Eom, C. H. Lange, C. B. Kim, R. B. Lauer, W. C. Rideout, and J. S. LaCourse, "Characterization of the dynamics of semiconductor lasers using optical modulation," IEEE J. Quantum Electron., vol. 28, pp. 118-127, 1992

[12] K. J. Vahala and M. A. Newkirk, "Parasitic-free modulation of semiconductor lasers," IEEE J. Quantum Electron., vol. 25, pp. 1393-1398, 1989.

[13] R. F. Nabiev, E. C. Vail, and C. J. Chang-Hasnain, "Temperature dependent efficiency and modulation characteristics of Al-free 980-nm laser diodes," IEEE J. Select. Topics Quantum Electron., vol. 1, pp. 234-243, 1995.

[14] N. Tessler, M. Margalit, R. Ben Michael, M. Orenstein, and G. Eisenstein, "Modulation of quantum well lasers by short optical excitation: Energy and spatial dependent effects," IEEE Photon. Technol. Lett., vol. 7, pp. 23-25, 1995.

[15] D. Vassilovski, T. C. Wu, S. Kan, K. Y. Lau, and C. E. Zah, "Unambiguous determination of quantum capture, carrier diffusion, and intrinsic effect in quantum-well laser dynamics using wavelengthselective optical modulation," IEEE Photon. Technol. Lett., vol. 7, pp. 706-708, 1995

[16] M. Grupen and K. Hess, "Simulation of carrier dynamics in quantum well lasers," Proc. SPIE, vol. 2693, pp. 374-385, 1996.

[17] M. Grupen and K. Hess, "Simulation of carrier transport and nonlinearities in quantum-well laser diodes," IEEE J. Quantum Electron., vol. 34, pp. 120-140, 1998.

[18] J. Minch, S. H. Park, T. Keating, and S. L. Chuang, "Theory and experiment of InGaAsP and InGaAlAs long-wavelength strained quantum-well lasers," IEEE J. Quantum Electron., vol. 35, pp. 771-782, May 1999.

[19] C. E. Zah, R. Bhat, B. N. Pathak, F. Favire, W. Lin, M. C. Wang, N C. Andreadakis, D. M. Hwang, M. A. Koza, T. P. Lee, Z. Wang, D. Darby, D. Flanders, and J. J. Hsieh, "High-performance uncooled 1.3$\mu \mathrm{m} \mathrm{AlGaInAs/InP} \mathrm{strained-layer} \mathrm{quantum-well} \mathrm{laser} \mathrm{for} \mathrm{subscriber} \mathrm{loop}$ applications," IEEE J. Quantum Electron., vol. 30, pp. 511-523, 1994.

[20] J. Shimizu, H. Yamada, S. Murata, A. Tomita, M. Kitamura, and A. Suzuki, "Optical-confinement-factor dependencies of the $K$ factor, differential gain, and nonlinear gain coefficient for $1.55 \mu \mathrm{m} \mathrm{In}$ GaAs/InGaAsP MQW and strained-MQW lasers," IEEE Photon. Technol. Lett., vol. 3, pp. 773-776, 1991.

[21] M. Ishikawa, R. Nagarajan, T. Fukushima, J. Wasserbauer, and J. Bowers, "Long wavelength high-speed semiconductor laser with carrier transport effects," IEEE J. Quantum Electron., vol. 28, pp. 2230-2241, 1992.

[22] S. Seki, K. Yokoyama, and P. Sotirelis, "Theoretical analysis of hightemperature characteristics of $1.3 \mu \mathrm{m}$ InP-based quantum-well lasers," IEEE J. Select. Topics Quantum Electron., vol. 1, pp. 264-274, 1995.

[23] S. Kakimoto and H. Watanabe, "Threshold current, differential gain, and relaxation resonance frequency of $1.55-\mu \mathrm{m}$ bulk and MQW DFB lasers diodes," IEEE J. Quantum Electron., vol. 34, pp. 1231-1239, 1998.

[24] W. Rideout, W. F. Sharfin, E. S. Koteles, M. O. Vassell, and B. Elman, "Well-barrier hole burning in quantum well lasers," IEEE Photon. Technol. Lett., vol. 3, pp. 784-786, 1991.

[25] S. J. Sweeney, A. F. Philips, A. R. Adams, E. P. O'Reilly, and P. J. A. Thijs, "The effect of temperature dependent processes on the performance of $1.5-\mu \mathrm{m}$ compressively strained $\mathrm{InGaAs}(\mathrm{P}) \mathrm{MQW}$ semiconductor diode lasers," IEEE Photon. Technol. Lett., vol. 10, pp. 1076-1078, 1998. 
[26] W. Fang, M. Hattendorf, S. L. Chuang, J. Minch, and C. S. Chang, "Analysis of temperature sensitivity in semiconductor lasers using gain and spontaneous emission measurements," Appl. Phys. Lett., vol. 70, pp. 796-798, 1997.

[27] L. F. Tiemeijer, P. J. A. Thijs, P. J. de Waard, J. J. M. Binsma, and T. V. Dogen, "Dependence of polarization, gain, linewidth enhancement factor, and $K$ factor on the sign of strain of InGaAs/InP strained-layer multiquantum well lasers," Appl. Phys. Lett., vol. 58, pp. 2738-2740, 1991.

[28] A. Yariv, "Scaling laws and minimum threshold currents for quantumconfined semiconductor lasers," Appl. Phys. Lett., vol. 53, pp 1033-1035, 1988.

[29] A. Haug, "Theory of the temperature dependence of the threshold current of an InGaAsP laser," IEEE J. Quantum Electron., vol. QE-21, pp. 716-718, 1985.

[30] H. Temkin, D. Coblentz, R. A. Logan, J. P. van der Ziel, T. Tanbun-Ek, R. D. Yadvish, and A. M. Sergent, "High temperature characteristics of InGaAsP/InP laser structures," Appl. Phys. Lett., vol. 62, pp. 2402-2404, 1993.

[31] Y. Zou, J. S. Osinski, P. Grodzinski, P. D. Dapkus, W. C. Rideout, W. F. Sharfin, J. Schlafer, and F. D. Crawford, "Experimental study of Auger recombination, gain, and temperature sensitivity of $1.5 \mu \mathrm{m}$ compressively strained semiconductor lasers," IEEE J. Quantum Electron., vol. 29, pp. 1565-1575, 1993.
[32] D. M. Byrne and B. A. Keating, "A laser diode model based on temperature dependent rate equations," IEEE Photon. Technol. Lett., vol. 1, pp. 1076-1078, 1989.

T. Keating, photograph and biography not available at the time of publication.

X. Jin, photograph and biography not available at the time of publication.

S. L. Chuang (S'78-M'82-SM'88-F'97), for biography, see p. 969 of the June 1999 issue of this JOURNAL.

K. Hess (M'79-SM'83-F'85), photograph and biography not available at the time of publication. 